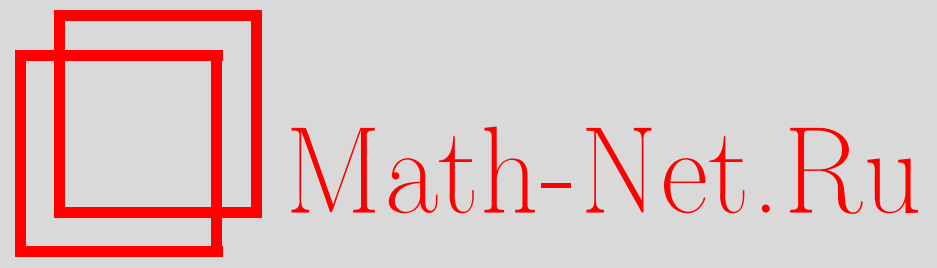

А. А. Сапоженко, О числе независимых множеств в расширителях, Дискрет. матем., 2001, том 13, выпуск 1, 56-62

DOI: https://doi.org/10.4213/dm276

Использование Общероссийского математического портала Math-Net.Ru подразумевает, что вы прочитали и согласны с пользовательским соглашением http://www. mathnet.ru/rus/agreement

Параметры загрузки:

IP: 54.197 .217 .227

26 апреля 2023 г., 17:51:41

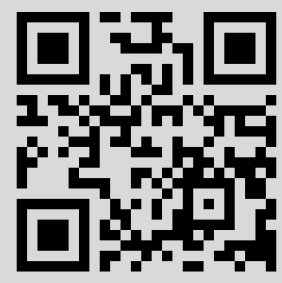


У ДК 519.1

\title{
О числе независимых множеств в расширителях
}

\author{
(C) 2001 г. A. А. Сапоженко
}

\begin{abstract}
Уточняется и обобщается на случай почти регулярных графов верхняя оценка для числа независимых множеств в регулярных графах, полученная Н. Алоном. Найдена верхняя оценка числа независимых множеств, размер которых существенно отличен от четверти числа вершин. Для числа независимых множеств в расширителях получена верхняя оценка вида $2^{n(1 / 2-c+o(1))}$, где $c$ положительная постоянная.

Работа выполнена при поддержке Российского фонда фундаментальных исследований, проект 01-01-00206.
\end{abstract}

\section{1. Введение}

В статье Н. Алона [1] получены верхние оценки вида $2^{n(1 / 2+o(1))}$ для числа независимых множеств в $n$-вершинных регулярных графах и в почти регулярных двудольных графах. Здесь получен более общий результат (см. теоремы 3 и 4) с более точной оценкой остаточного члена. Для регулярных графов степени $k$ различие состоит в том, что вместо $O\left(k^{-1 / 10}\right)$ в [1] здесь стоит $O(\sqrt{(\log k) / k})$ (здесь и далее используется логарифмы по основанию 2). Эта оценка остаточного члена неулучшаема по порядку, если разность между максимальной и минимальной степенями не меньше $\sqrt{k \log k}$. Отметим, что для регулярных графов она все еще значительно отличается от нижней оценки вида $O(1 / k)$, полученной в [1]. Кроме того, доказано (см. теорему 5), что в почти регулярных графах с $n$ вершинами число тех независимых множеств, мощность которых сильно отличается от $n / 4$, мало по сравнению с $2^{n / 2}$. Это следует из того, что во всяком почти регулярном графе небольшое число подмножеств мощности, приблизительно равной $n / 2$, содержит почти все независимые множества.

Основным результатом статьи является получение для числа независимых множеств в $\delta$-расширителях верхней границы вида $2^{n(1 / 2-c \delta+o(1))}$. Эта оценка позволяет получать (см., например, [3]) асимптотику исследуемой величины там, где с помощью оценок вида $2^{n(1 / 2+o(1))}$ для числа независимых множеств получается лишь асимптотика ее логарифма.

Ранее в [2] была найдена асимптотика числа независимых множеств в $n$-мерном кубе. В статье автора [4] (см. также [5]) получены оценки для числа независимых множеств в двудольных графах. Здесь подобный результат доказывается для произвольных (не двудольных) графов. Оценки получены методами, использовавшимися в статьях [6] и [7]. 


\section{2. Определения и формулировка результата}

Рассматриваются графы, не содержащие кратных ребер и петель. Вершины графов пронумерованы. Граф с $n$ вершинами, в котором минимальная степень вершины равна $k$, а максимальная не превосходит $k+\theta$, назовем $(n, k, \theta)$-графом. Везде в дальнейшем предполагается, что $n$ и $k$ достаточно велики, а $\theta=o(k)$ при $k \rightarrow \infty$. При выполнении последнего условия $(n, k, \theta)$-граф называется почти регулярным.

Подмножество $A$ вершин графа $G$ называется независимым множеством, если подграф, порожденный подмножеством $A$, не содержит ребер. Семейство всех независимых множеств графа $G$ обозначим через $\mathscr{I}(G)$ и положим $I(G)=|\mathscr{I}(G)|$. Пусть $G=(V ; E)$ - граф с множеством вершин $V$ и множеством ребер $E$, а $v \in V$. Назовем границей вершины $v$ в графе $G$ множество

$$
\partial(v)=\{u:(u, v) \in E\} .
$$

Ясно, что $\sigma(v)=|\partial(v)|$ есть степень вершины $v$. Границу подмножества $A$ вершин графа $G$, определим как множество

$$
\partial(A)=\bigcup_{v \in A} \partial(v) \backslash A .
$$

Пусть $0 \leqslant \delta<1$. Граф $G=(V ; E)$ назовем $\delta$-расширителем, если $|A| \leqslant|\partial(A)|(1-\delta)$ для всех $A \in \mathscr{I}(G)$. Основным результатом является следующая теорема.

Теорема 1. Пусть $G=(V ; E)$ лвляетсл $(n, k, \theta)$-графом $u \delta$-расширителем при некотором $0 \leqslant \delta<1$. Тогда

$$
I(G) \leqslant 2^{(1 / 2) n(1-\delta / 7+O(\theta / k+\sqrt{(\log k) / k}))} .
$$

Из теоремы 1 при $\theta=0$ получаем следующее утверждение.

Теорема 2. Пусть п-вершинный регулярный граф $G=(V ; E)$ степени $k$ является $\delta$-расширителем при некотором $0 \leqslant \delta<1$. Тогда

$$
I(G) \leqslant 2^{(1 / 2) n(1-\delta / 7+O(\sqrt{(\log k) / k)})} .
$$

\section{3. Вспомогательные утверждения}

Лемма 1. Пусть $G=(V ; E)$ лвляетсл $(n, k, \theta)$-графом, $0<\varphi<k u A \subseteq V$ является независимым множеством. Тогда существует множество $T \subseteq A$ такое, чmo

$$
\begin{aligned}
|T| & \leqslant|\partial(A)| / \varphi, \\
A \subseteq D & =D(T)=\{v \in V \backslash \partial(A):|\partial(v) \backslash \partial(T)|<\varphi\}, \\
|D| & \leqslant|\partial(T)| \frac{k+\theta}{k-\varphi} .
\end{aligned}
$$

Доказателъство. Множество $T$ построим с помощью следующей пошаговой процедуры. Пусть $u_{1}$ - произвольная вершина из $A$. На первом шаге положим $T_{1}=\left\{u_{1}\right\}$. Пусть сделано $m$ шагов и построено множество

$$
T_{m}=\left\{u_{1}, \ldots, u_{m}\right\}
$$


Если существует вершина $u_{m+1} \in A$ такая, что $\left|\partial\left(u_{m+1}\right) \backslash \partial\left(T_{m}\right)\right| \geqslant \varphi$, то на шаге $m+1$ полагаем

$$
T_{m+1}=T_{m} \cup\left\{u_{m+1}\right\} .
$$

В противном случае процесс заканчивается и полагаем $T=T_{m}$.

Неравенство (3) и включение (4), очевидно, выполняются для построенного $T$. Неравенство (5) следует из того, что $|\partial(v) \cap \partial(T)| \geqslant k-\varphi$ для всех $v \in D$ и $|\partial(u)| \leqslant$ $k+\theta$ для всех $u \in \partial(T)$.

Из леммы 1 вытекают оценки для мощности типичных независимых множеств и их числа. Множество $T$, удовлетворяющее условиям леммы 1 , будем называть $\varphi$-локализатором множества $A$. Пусть $G-n$-вершинный граф с минимальной степенью вершины, равной $k$. Везде в дальнейшем будем полагать, что

$$
\varphi=\sqrt{k \log k}
$$

Семейство множеств $A \in \mathscr{I}(G)$, для которых множество $T$ является $\varphi$-локализатором, обозначим через $\mathscr{I}(G, T)$. Пусть $I(G, T)=|\mathscr{I}(G, T)|$.

Теорема 3. Пустъ $G=(V ; E)$ является $(n, k, \theta)$-графом. Тогда

$$
I(G) \leqslant 2^{(1 / 2) n(1+O(\theta / k+\sqrt{(\log k) / k}))} .
$$

Доказательство. Пусть $T$ является $\varphi$-локализатором множества $A$ в графе $G$, множество $D=D(T)$ определено в соответствии с (4). Заметим, что

$$
|D| \leqslant(n / 2)(k+\theta) /(k-\varphi),
$$

ибо в противном случае в силу (5)

$$
|\partial(T)| \geqslant|D|(k-\varphi) /(k+\theta)>n / 2,
$$

а, следовательно, $|\partial(T)|+|D|>n$, что противоречит условию $\partial(T) \cap D=\varnothing$.

Оценим теперь число $\varphi$-локализаторов при $\varphi=\sqrt{k \log k}$. С использованием неравенства (3) и того, что $|\partial(A)| \leqslant n$ для любого $A$, находим, что

$$
\sum_{i \leqslant n / \varphi}\left(\begin{array}{l}
n \\
i
\end{array}\right) \leqslant 2^{n \sqrt{(\log k) / k}}
$$

при этом использовалась оценка

$$
\sum_{i \leqslant m}\left(\begin{array}{l}
n \\
i
\end{array}\right) \leqslant(e n / m)^{m}
$$

(см., например, [7]). Теперь, поскольку в силу (4) и (7)

$$
I(G, T) \leqslant 2^{|D|} \leqslant 2^{(1 / 2) n(1+O(\theta / k+\sqrt{(\log k) / k}))},
$$

с учетом (8) получаем оценку

$$
I(G) \leqslant \sum_{T \subseteq V,|T| \leqslant n / \varphi} I(G, T) \leqslant 2^{(1 / 2) n(1+O(\theta / k+\sqrt{(\log k) / k}))} .
$$


При $\theta=0$ в (10) получаем теорему 1.1 из [1] с лучшей оценкой остаточного члена.

Теорема 4. Для любого $n$-вершинного регулярного графа $G$ степени $k$

$$
I(G) \leqslant 2^{(1 / 2) n(1+O(\sqrt{(\log k) / k}))} .
$$

Теорема 5. Пусть $G=(V ; E)$ являетсл $(n, k, \theta)$-графом. Пусть $0<\beta<1 u I_{\beta}(G)$ - число тех $A \in \mathscr{I}(G)$, для которых ||$A|-n / 4| \geqslant \beta n / 4$. Тогда

$$
I_{\beta}(G) \leqslant 2^{(1 / 2) n\left(1-\beta^{2} /(2 \ln 2)+O(\theta / k+\sqrt{(\log k) / k})\right)} .
$$

Доказательство. Для $0<\beta<1$ и $T \subseteq V$ пусть

$$
\mathscr{I}_{\beta}(G, T)=\{A \in \mathscr{I}(G, T):|| A|-n / 4| \geqslant \beta n / 4\} .
$$

Пусть $D=D(T)$ определено множеством $T$ в соответствии с (4). Разобьем $\mathscr{I}_{\beta}(G, T)$ на два подсемейства

$$
\begin{aligned}
& \mathscr{I}_{1, \beta}(G, T)=\left\{A \in \mathscr{I}_{\beta}(G, T):|D|>n / 2\right\}, \\
& \mathscr{I}_{2, \beta}(G, T)=\mathscr{I}_{\beta}(G, T) \backslash \mathscr{I}_{1, \beta}(G, T) .
\end{aligned}
$$

Положим $d=|D|$. Если $d>n / 2$, то

$$
d=(n / 2)(1+O(\theta / k+\sqrt{(\log k) / k}))
$$

ввиду (7). Поэтому из неравенства ||$A|-n / 4| \geqslant \beta n / 4$ вытекает, что

$$
|| A|-d / 2| \geqslant(d / 2)(\beta+\gamma)
$$

где

$$
\gamma=O(\theta / k+\sqrt{(\log k) / k})
$$

для любого $A \in \mathscr{I}_{1, \beta}(G, T)$.

Пусть $0<x<1$. Из неравенства для больших уклонений (см., например, [8]) следует, что число подмножеств $A \subseteq D$ таких, что ||$A|-d / 2| \geqslant x d / 2$, не превышает $2 \cdot 2^{d\left(1-x^{2} /(2 \ln 2)\right.}$. В силу сказанного выше

$$
\begin{aligned}
\mathscr{I}_{1, \beta}(G, T) & \leqslant \sum_{|i-d / 2| \geqslant(d / 2)(\beta+\gamma)}\left(\begin{array}{l}
d \\
i
\end{array}\right) \leqslant 2 \cdot 2^{d\left(1-(\beta+\gamma)^{2} /(2 \ln 2)\right)} \\
& \leqslant 2^{(1 / 2) n\left(1-\beta^{2} /(2 \ln 2)+O(\theta / k+\sqrt{(\log k) / k})\right)}
\end{aligned}
$$

С использованием (4) и неравенства $d \leqslant n / 2$ получаем, что

$$
\left|\mathscr{I}_{2, \beta}(G, T)\right| \leqslant \sum_{|i-n / 4| \geqslant \beta n / 4}\left(\begin{array}{l}
d \\
i
\end{array}\right) \leqslant \sum_{|i-n / 4| \geqslant \beta n / 4}\left(\begin{array}{c}
n / 2 \\
i
\end{array}\right) \leqslant 2 \cdot 2^{(1 / 2) n\left(1-\beta^{2} /(2 \ln 2)\right)}
$$

Из (13) и (14) следует, что

$$
\left|\mathscr{I}_{\beta}(G, T)\right| \leqslant\left|\mathscr{I}_{1, \beta}(G, T)\right|+\left|\mathscr{I}_{2, \beta}(G, T)\right| \leqslant 2^{(1 / 2) n\left(1-\beta^{2} /(2 \ln 2)+O(\theta / k+\sqrt{(\log k) / k})\right.} .
$$

Из (15) аналогично (10) вытекает (12). 
Из теоремы 5 при $\theta=0$ вытекает следующая теорема.

Теорема 6. Пусть $0<\beta<1 u I_{\beta}(G)$ - число тех $A \in \mathscr{I}(G)$, для которых ||$A|-n / 4| \geqslant \beta n / 4$. Тогда для любого $n$-вершинного регулярного графа $G$ степени $k$

$$
I_{\beta}(G) \leqslant 2^{(1 / 2) n\left(1-\beta^{2} /(2 \ln 2)+O(\sqrt{(\log k) / k})\right)} .
$$

Подграф графа $G$ называется остовным, если он содержит все вершины графа $G$. Звездой называется связный двудольный граф, в котором хотя бы одна из долей вершин состоит ровно из одной вершины. Вершину максимальной степени в звезде назовем центром этой звезды. В случае, когда звезда представляет собой ребро, центром ее будет считаться та вершина, которая имеет наименьший номер. Степенью звезды назовем максимальную из степеней ее вершин. Назовем лес звездным, если каждая компонента связности является звездой.

Лемма 2. Если $F$ является звездным лесом со степенями компонент связности $\lambda_{1}, \ldots, \lambda_{t}, m o$

$$
I(F)=\prod_{1 \leqslant i \leqslant t}\left(2^{\lambda_{i}}+1\right) .
$$

Доказателъство. Число независимых множеств (включая пустое множество) звезды степени $\lambda$ равно, очевидно, $2^{\lambda}+1$. Равенство (17) следует теперь из того, что число независимых множеств графа равно произведению чисел независимых множеств его компонент связности.

Лемма 3. Во всяком графе $G$ без изолированных вершин можно выделить остовнъй звездный лес.

Доказательство. Известно, что в графе без изолированных вершин можно выделить остовный лес. Поэтому достаточно показать, что во всяком дереве можно выделить остовный звездный лес. Если дерево не является звездой и отлично от изолированной вершины, то в нем существует вершина такая, что все инцидентные ей ребра, кроме ровно одного, являются висячими (инцидентными вершине степени 1). Удалим ребро, не являющееся висячим. Получим остовный лес с большим, чем у прежнего, числом вершин, содержащихся в компонентах связности, являющихся звездами. Продолжая процедуру удаления ребер, построим остовный звездный лес.

Замечание 1. Если вершины графа пронумерованы, то можно считать, что все его остовные звездные леса также пронумерованы и графу однозначно сопоставлен его остовный звездный лес (например, первый в принятой нумерации).

Независимое множество $A$ графа $G=(V ; E)$ назовем максимальным, если $A \cup$ $\partial(A)=V$. Семейство всех максимальных множеств графа $G$ обозначим через $\mathscr{M}(G)$. Положим

$$
\mathscr{M}(G, a)=\{A \in \mathscr{M}(G):|A|=a\} .
$$

Замечание 2. Каждое независимое множество содержится в некотором максимальном. 
Лемма 4. Если $G$ является $n$-вершинным $\delta$-расширителем $и A$ - независимое множество графа $G$, то

$$
|A| \leqslant n(1-\delta) /(2-\delta)
$$

Доказателъство. Из определения $\delta$-расширителя и независимости $A$, получаем, что

$$
|A| \leqslant|\partial(A)|(1-\delta) \leqslant(n-|A|)(1-\delta) .
$$

Отсюда следует (18).

\section{4. Доказательство теоремы 1}

Как и при доказательстве теоремы 3 , оценим сначала $I(G, T)$. Обозначим через $I(G, T, a)$ число множеств $B \in \mathscr{I}(G, T)$ таких, что $B \subseteq A$ для некоторого $A \in \mathscr{M}(G, a) \cap \mathscr{I}(G, T)$. В силу замечания 1 и (18)

$$
I(G, T) \leqslant \sum_{a \leqslant n(1-\delta) /(2-\delta)} I(G, T, a) .
$$

Пусть $T \subseteq V$ является $\sqrt{k \log k}$-локализатором хотя бы для одного $A \in \mathscr{M}(G, a)$. Согласно лемме 1 множество $T$ однозначно определяет свою границу $\partial(T)$ в графе $G=(V ; E)$ и множество $D$ такое, что $A \subseteq D$ для всякого $A \in \mathscr{I}(G, T)$. Пусть $U=V \backslash(D \cup \partial(T))$. Ясно, что $U$ также однозначно определяется множеством $T$. Заметим, что $U \subseteq \partial(A)$ для всякого $A \in \mathscr{M}(G) \cap \mathscr{I}(G, T)$, поскольку

$$
U \subseteq V \backslash D \subseteq V \backslash A=\partial(A) .
$$

Пусть подграф $\Gamma=\left(D ; E^{\prime}\right)$ графа $G$ порожден множеством $D$. Обозначим через $Q$ подмножество тех $v \in D$, которые не являются изолированными в графе $\Gamma$. Через $\Gamma^{\prime}=\left(Q ; E^{\prime \prime}\right)$ обозначим подграф графа $\Gamma$, порожденный множеством $Q$. Пусть далее $F$ является остовным звездным лесом графа $\Gamma^{\prime}=\left(Q ; E^{\prime \prime}\right)$. В силу замечания 1 лес $F$ определен однозначно графом $\Gamma^{\prime}=\left(Q ; E^{\prime \prime}\right)$, а значит, и множеством $T$.

Пусть $|Q|=q$, а лес $F$ состоит из $t$ компонент связности, степенями которых являются числа $\lambda_{1}, \ldots, \lambda_{t}$. Заметим, что множество $A \in \mathscr{M}(G, a) \cap \mathscr{I}(G, T)$ определено однозначно, если для центра каждой из звезд леса $F$ известно, какому из множеств $A$ или $\partial(A)$ принадлежит этот центр. Число возможностей не превосходит $2^{t}$. Число подмножеств множества $A$ из $\mathscr{M}(G, a)$ равно $2^{a}$. Поэтому

$$
I(G, T, a) \leqslant 2^{t+a} .
$$

Из (20) и (21) получаем, что

$$
I(G, T) \leqslant \sum_{a \leqslant n(1-\delta) /(2-\delta)} 2^{t+a} \leqslant 2^{1+t+n(1-\delta) /(2-\delta)} .
$$

Заметим, что в силу (4) множество $B \in \mathscr{I}(G, T)$ определено, если заданы множества $B \cap Q$ и $B \cap(D \backslash Q)$. Полагая $|D|=d$, с использованием (17) получаем, что

$$
I(G, T) \leqslant I(F) 2^{|D \backslash Q|} \leqslant 2^{d-q} \prod_{1 \leqslant i \leqslant t}\left(2^{\lambda_{i}}+1\right) .
$$


Заметим, что

$$
\prod_{1 \leqslant i \leqslant t}\left(2^{\lambda_{i}}+1\right)=2^{q-t} \prod_{1 \leqslant i \leqslant t}\left(1+2^{-\lambda_{i}}\right) \leqslant 2^{q-t+t \log (3 / 2)} .
$$

Из (23) и (24) следует, что

$$
I(G, T) \leqslant 2^{d-t(1-\log (3 / 2))} \leqslant 2^{d-2 t / 5} .
$$

Если $t \leqslant 5 n \delta /(14(2-\delta))$, то из неравенств $0 \leqslant \delta<1$ и (22) следует, что

$$
I(G, T) \leqslant 2^{1+(1 / 2) n(1-2 \delta /(7(2-\delta)))} \leqslant 2^{(1 / 2) n(1-\delta / 7+O(1 / n))} .
$$

Если $t>5 n \delta /(14(2-\delta))$, то из (25) и (7) следует, что

$$
I(G, T) \leqslant 2^{(1 / 2) n(1-\delta / 7+O(\theta / k+\sqrt{(\log k) / k}))} .
$$

Теперь (1) получается из (8), (26) и (27) суммированием по $T$ аналогично тому, как

(6) получено из (8) и (9).

\section{Список литературы}

1. Alon N., Independent sets in regular graphs and sum-free subsets of finite groups. Israel $J$. Math. (1991) 73, №2, 247-256.

2. Коршунов А. Д., Сапоженко А. А., О числе двоичных кодов с расстоянием 2. Пробл. кибернетики (1983) 40, 111-140.

3. Sapozhenko A. A., On the number of independent sets in graphs and the number of sum-free sets in abelian groups. Материалы Международной конференции "Дискретный анализ и исследование операчий”, 26 июня - 1 июля, 2000, с. 89.

4. Сапоженко А. А., О числе независимых множеств в двудольных графах. Tруды IV международной конференции “Дискретнье модели в теории управляющих систем", 19-25 июня 2000, Москва, МАКС Пресс, с. 116-119.

5. Sapozhenko A. A., On the number of independent sets in bipartite graphs with large minimum degree. DIMACS Technical Report 2000-25, August 2000.

6. Сапоженко А. А., О числе связных подмножеств с заданной мощностью границы в двудольных графах. Методы дискретного анализа в решении комбинаторных задач (1987) 45, 42-70.

7. Сапоженко А. А., О числе связных подмножеств с заданной мощностью окрестности в графе. Дискретный анализ и исследование операций (1997) 4, №3, 18-34.

8. Ширяев А. Н., Вероятностъ. Наука, Москва, 1989.

Статья поступила 15.12.2000. 